\title{
Soft QCD in ATLAS: Minimum bias and diffraction studies ${ }^{1}$
}

\author{
Edward K. Sarkisyan-Grinbaum ${ }^{\S, \ddagger}$ \\ (for the ATLAS Collaboration) \\ §University of Texas at Arlington, Department of Physics, Arlington, TX 76019, USA \\ ${ }^{\ddagger}$ Department of Physics, CERN, CH-1211 Geneva 23, Switzerland
}

\begin{abstract}
We present measurements of charged particle production in proton-proton collisions at centre-of-mass energies of $\sqrt{s}=0.9,2.36$ and $7 \mathrm{TeV}$ recorded with the ATLAS detector at the Large Hadron Collider. Events were collected using a single-arm minimum bias trigger, charged tracks are measured with high precision in the inner tracking system. Minimum bias analysis uses data samples at all three energies, while diffractive events are studied using a sample of events at $\sqrt{s}=7 \mathrm{TeV}$. To study diffractive interactions, the events that have hits on exactly one side of the ATLAS detector were selected. The charged particle multiplicity, pseudorapidity and transverse momentum spectra are analyzed and compared to the predictions by various Monte Carlo models.
\end{abstract}

\section{Introduction}

The results of soft QCD measurements of charged particle production in protonproton collisions at centre-of-mass energies of $\sqrt{s}=0.9,2.36$ and $7 \mathrm{TeV}$ using the ATLAS detector [1] at the LHC are presented here. The soft, or non-perturbative, QCD processes of multiparticle production include diffractive (single- and doublediffractive) and non-diffractive components forming the inelastic scattering. All these ingredients are not well modeled by perturbative QCD or Monte Carlo (MC) models when applied to low transverse momentum, or soft, particle production [2]. The MC models need to be (re)tuned to the data. An understanding of soft particle production processes is important for precision (high transverse momentum) measurements to be made at the LHC and is crucial for understanding of QCD effects, total cross section determination, understanding of saturation effects, jet studies, mass reconstruction. These measurements should be done early on, at low luminosity to avoid the effect of overlapping ("pile-up") collisions at high luminosities. We present minimum bias [3,4] and diffractive [5] measurements, while another report presents studies of azimuthal spectra and underlying events [6].

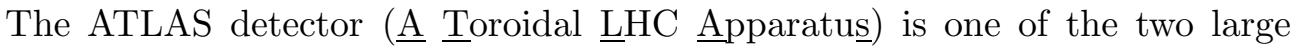
general propose detectors at the LHC. For the measurements presented here, the trigger system and the tracking devices were of particular importance. The ATLAS

\footnotetext{
${ }^{1}$ Invited talk at the VI International Workshop "Particle Correlations and Femtoscopy", Bogolyubov Institute for Theoretical Physics, Kiev, Ukraine, September 14 - 18, 2010.
} 
Inner Detector (ID) immersed in a 2 Tesla axial magnetic field is used to measure charged particles. It consists of three sub-detectors: pixel detector (Pixel), a silicon strip detector (SCT) and a transition radiation tracker. The inner detector has a full coverage ${ }^{2}$ in the azimuthal angle and covers the pseudorapidity range $|\eta|<2.5$. $^{2}$

The ATLAS trigger system is a three-level trigger system. For the measurements presented here, the trigger relies on the Beam Pickup Timing devices (BPTX) and the Minimum Bias Trigger Scintillators (MBTS). The BPTX are composed of electrostatic beam pick-ups attached to the beam pipe at $\pm 175 \mathrm{~m}$ from the center of the ATLAS detector. The MBTS are mounted at each end of the detector in front of the liquid-argon end-cap calorimeter cryostats at $z= \pm 3.56 \mathrm{~m}$ and are segmented into eight sectors (16 independent wedge-shaped plastic scintillators) in azimuth and in two rings in pseudorapidity $(2.09<|\eta|<2.82$ and $2.82<|\eta|<3.84)$. Data were taken for these measurements using the singlearm MBTS trigger, formed from BPTX and MBTS triggers. The MBTS trigger efficiency was studied with a separate prescaled Level-1 BPTX trigger, filtered to obtain inelastic interactions by ID requirements at higher level triggers. The MBTS efficiency was found to be $>99 \%$. The two MBTS time measurements were used to veto halo and beam-gas events, as well as a gap trigger for diffractive studies, as described below.

The data at $\sqrt{s}=900 \mathrm{GeV}$ and $2.36 \mathrm{TeV}$ have been recorded in December 2009 and the $7 \mathrm{TeV}$ data, used here, have been recorded in March-April 2010. During the $2.36 \mathrm{TeV}$ data collection, stable conditions were not declared. Therefore, ensure detector safety, the SCT was in standby mode with lower hit efficiencies and increased noise. To estimate track reconstruction efficiency, two complementary methods were developed [4] to measure the spectra: the first used the full ID information and corrected the efficiency from the simulation using a data-driven technique, while the second used tracks reconstruction from Pixel information only. The results obtained by these methods were found to agree within $3 \%$.

\section{Data and Monte Carlo}

\subsection{Data selection}

The data used here comprise about $357 \mathrm{~K}$ selected events at $\sqrt{s}=900 \mathrm{GeV}$, about $10 \mathrm{M}$ events at $\sqrt{s}=7 \mathrm{TeV}$, and about $6 \mathrm{~K}$ events at $\sqrt{s}=2.36 \mathrm{TeV}$. These data

\footnotetext{
${ }^{2}$ The ATLAS reference system is a Cartesian right-handed co-ordinate system, with the nominal collision point at the origin. The anti-clockwise beam direction defines the positive $z$-axis, while the positive $x$-axis is defined as pointing from the collision point to the centre of the LHC ring and the positive $y$-axis points upwards. The azimuthal angle $\phi$ is measured around the beam axis, and the polar angle $\theta$ is measured with respect to the $z$-axis. The pseudorapidity is defined as $\eta=-\ln \tan (\theta / 2)$, and $p_{T}$ is the momentum component transverse to the beam direction.
} 

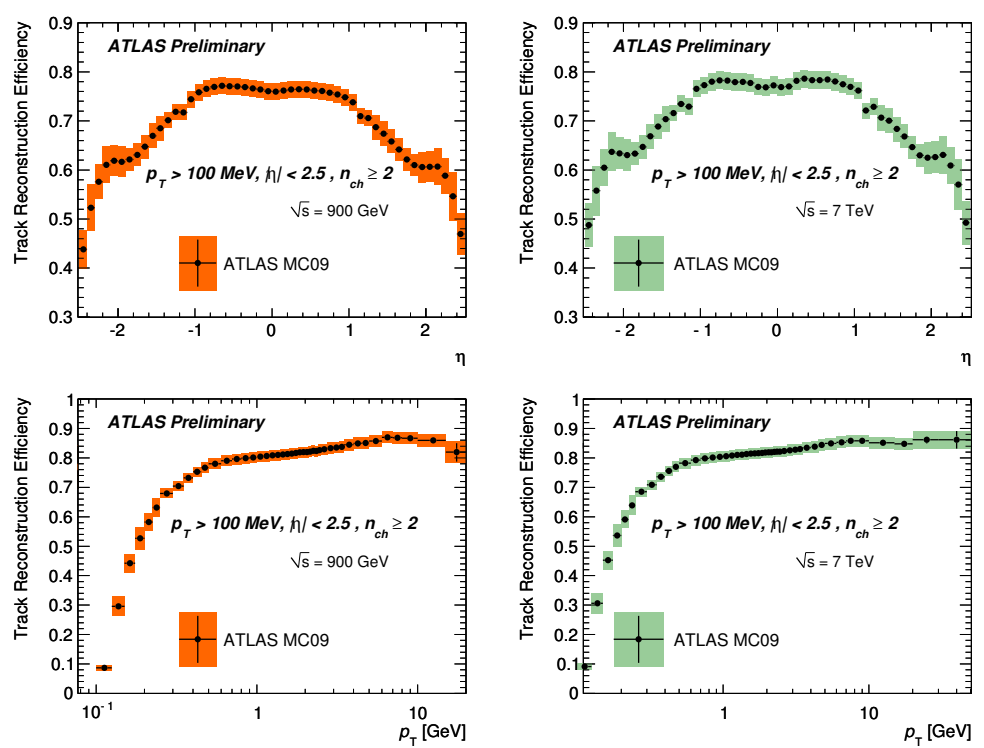

Figure 1: The track reconstruction efficiency as a function of $\eta$ (upper panel) and $p_{T}$ (bottom) as derived from PYTHIA6 tune MC09 (Sect. 2.1). The statistical errors are shown as black lines, the total errors as colour shaded areas. The distributions are shown at $\sqrt{s}=900 \mathrm{GeV}$ (left plots) and $7 \mathrm{TeV}$ (right plots).

samples correspond to integrated luminosities of approximately $7 \mu \mathrm{b}^{-1}, 0.1 \mu \mathrm{b}^{-1}$ and $190 \mu \mathrm{b}^{-1}$ at $\sqrt{s}=0.9,2.36$ and $7 \mathrm{TeV}$, respectively.

To reduce the contribution from background events and non-primary tracks, as well as to minimize the systematic uncertainties, the events were required: to have fired a single-arm Level-1 MBTS trigger, the primary vertex (PV) to be reconstructed using the beam-spot (BS) information, to not have a second primary vertex with four or more tracks in the same bunch crossing (to remove pile-up), and to have at least two good tracks in the event. A good track then is defined as one that satisfies: transverse momentum $p_{T}>100 \mathrm{MeV}(>500 \mathrm{MeV}$ at $\sqrt{s}=2.36 \mathrm{TeV})$ and $|\eta|<2.5$; a hit in the first layer of the Pixel detector; a minimum of one Pixel hit in any of the 3 layers; at least two $\left(p_{T}>100 \mathrm{MeV}\right)$, four $\left(p_{T}>200 \mathrm{MeV}\right)$ or six $\left(p_{T}>300 \mathrm{MeV}\right) \mathrm{SCT}$ hits $N_{\text {sct }}$ at $\sqrt{s}=0.9$ and $7 \mathrm{TeV}$, and $N_{\text {sct }}>6$ at $\sqrt{s}=2.36 \mathrm{TeV}$; the transverse and longitudinal impact parameters relative the PV: $\left|d_{0}\right|<1.5 \mathrm{~mm},\left|z_{0} \cdot \sin (\theta)\right|<1.5 \mathrm{~mm}$, respectively. The residual backgrounds are found to be small or negligible: cosmic ray and beam background $<10^{-6}$ and $<0.1 \%$, respectively, and pileup contribution of the order of $0.01 \%$.

Track reconstruction efficiencies were determined from MC (Sect. 2.2) using full detector simulation and reconstruction. A key issue is the description of material in the tracking volume. This was tuned to match the data. The track reconstruction efficiency is studied as a function of $\eta$ and $p_{T}$, as shown in Fig. 1 for 0.9 and $7 \mathrm{TeV}$. The difference in the track reconstruction efficiencies between 

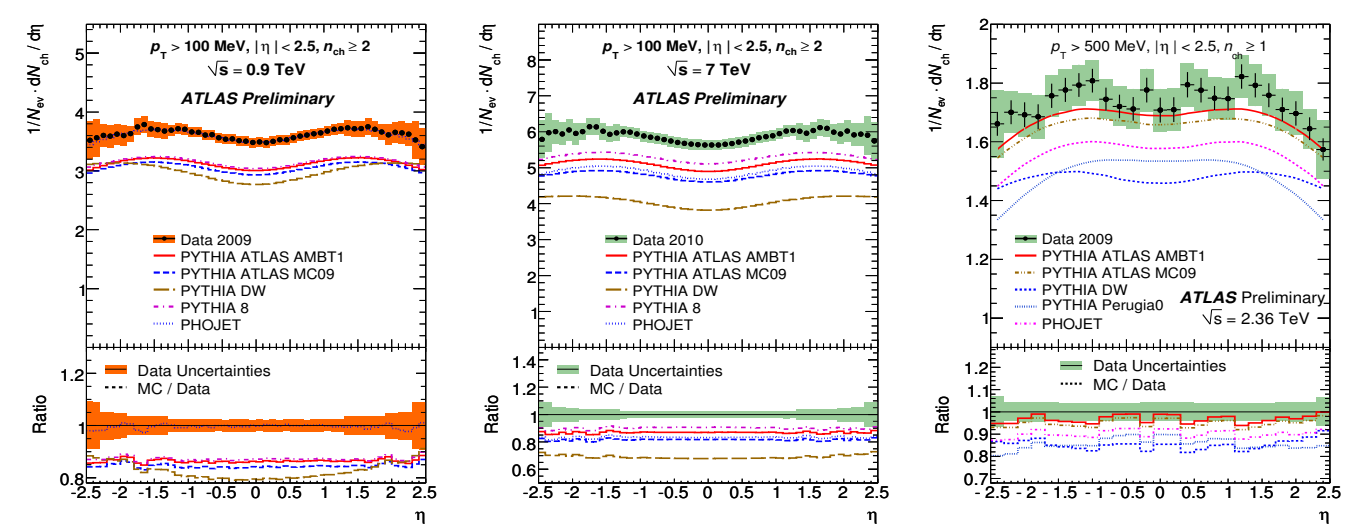

Figure 2: Charged-particle $\eta$ spectrum at $\sqrt{s}=0.9,7$ and $2.36 \mathrm{TeV}$. The distribution at $2.36 \mathrm{TeV}$ is for $p_{T}>500 \mathrm{MeV}$ and with at least one track. The values of the ratio histograms refer to the bin centroids. The data are shown the same way as in Fig. 1.

two energies is dominated by different number and configuration of the disabled Pixel and SCT modules in the running conditions. The trigger, vertex and tracking efficiencies were used to correct the data back to particle level $[3,4,7]$. Where possible the data were used to reduce MC dependencies. More details on the determination of efficiencies, backgrounds, uncertainties and correction procedure can be found in $[3,4,7]$. It should be noted that no corrections applied in the diffraction analysis [5].

\subsection{Monte Carlo models}

The PYTHIA [8] MC generator was used as a main generator in the ATLAS studies. The parameters of this generator have been tuned to describe chargedhadron production and the underlying event in $\mathrm{pp}$ and $\mathrm{p} \overline{\mathrm{p}}$ data at energies 0.2 to $1.96 \mathrm{TeV}$. Samples of single-diffractive, double-diffractive and non-diffractive events were produced using the PYTHIA6 (6.4.21) generator. A specific set of optimized parameters, the ATLAS MC09 PYTHIA6 tune [9], is the reference tune throughout these studies. These parameters were derived by tuning to underlying event and minimum bias data from Tevatron at 0.63 and $1.8 \mathrm{TeV}$. The $\mathrm{MC}$ samples generated with this tune were used to determine detector acceptances and efficiencies and to correct the data. The diffractive and non-diffractive contributions in the generated samples were mixed according to the generator cross sections to describe the inelastic scattering. All the events were processed through the ATLAS detector simulation code [10].

For the purpose of comparing the present measurement to different phenomenological models describing minimum-bias events, the following additional PYTHIA6 samples were generated: the ATLAS data-based AMBT1 tune [11], which was ob- 

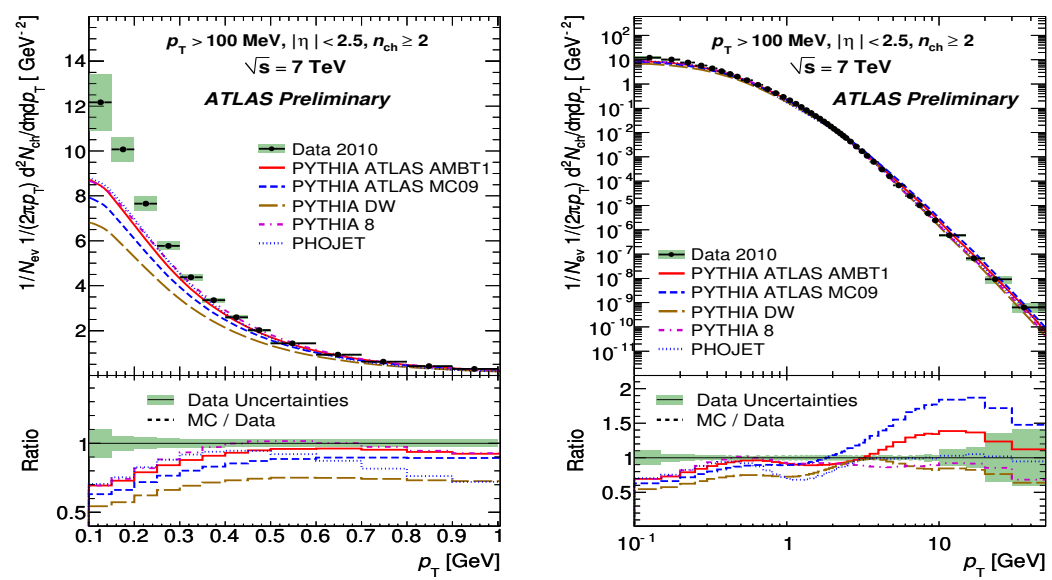

Figure 3: Charged-particle $p_{T}$ spectrum at $\sqrt{s}=7 \mathrm{TeV}$. The left panel shows the distribution in linear scale up to $p_{T}=1 \mathrm{GeV}$, and the right panel shows the full-range distribution in log-log scale. The data are shown the same way as in Fig. 2.

tained by attempting to fit charged particle multiplicity distributions in a diffraction limited phase-space; the Perugia0 tune [12], in which soft-QCD part is tuned using only minimum-bias data from the Tevatron and CERN p $\bar{p}$ colliders; the DW tune [13], which uses the virtuality-ordered showers and was derived to describe the CDF Run II underlying event and Drell-Yan data. In addition, the PYTHIA8 generator [14] was used which includes new features such as hard scattering in diffractive systems, up-to-date parton density function (pdf) set, a possibility to use one pdf set for hard scattering and another for the rest. Finally, the PHOJET generator [15] used as an alternative model where soft processes use Pomeron exchange and semi-hard processes are described by perturbative parton scattering.

\section{Minimum bias measurements}

Figure 2 shows the pseudorapidity distributions measured at $\sqrt{s}=0.9$ and $7 \mathrm{TeV}$. The measurements are 5 to $20 \%$ higher than the MC predictions, except the $900 \mathrm{GeV}$ PHOJET prediction is on top of the data. Interestingly, in both cases, PYTHIA8 is near to the data, being even closer than the AMBT1 model at $7 \mathrm{TeV}$. At $2.36 \mathrm{TeV}$, where the distribution is measured at $p_{T}>500 \mathrm{MeV}$, the AMBT1 and MC09 tunes slightly underestimate the data, while other MC models are lower by $10-20 \%$ than the data.

Figures 3 and 4 show, respectively, $p_{T}$ and multiplicity $n_{\mathrm{ch}}$ distributions of charged particles detected at $\sqrt{s}=7 \mathrm{TeV}$. The MC $p_{T}$ spectra agree well with the data at intermediate $p_{T}$ from 0.5 to $2 \mathrm{GeV}$, while largely disagree at lower and higher $p_{T}$. The data differs from MC predictions at low miltiplicities, and at $n_{\mathrm{ch}}>40$ PYTHIA tunes underestimate the data by $50 \%$. Similar observations are 

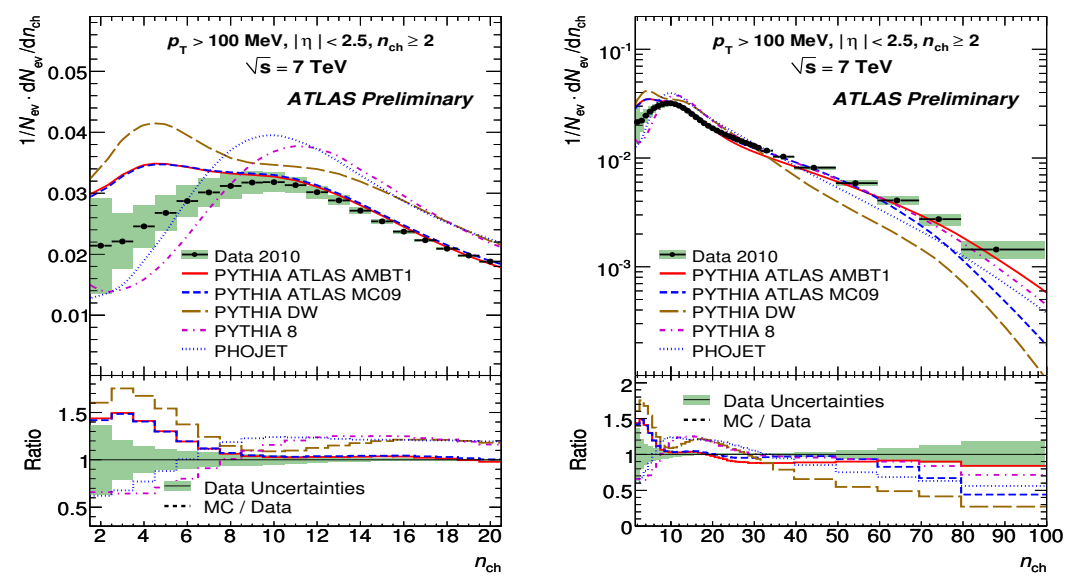

Figure 4: Charged-particle multiplicities at $\sqrt{s}=7 \mathrm{TeV}$. The left panel shows the distribution in linear scale up to $n_{\mathrm{ch}}=20$, and the right panel shows the full-range distribution in $\log$ scale. The data are shown the same way as in Fig. 2.

made from $900 \mathrm{GeV}$ studies and earlier studies at $p_{T}>500 \mathrm{MeV}$, as well as from measurements at $2.36 \mathrm{TeV}$. The deviation is expected due to contribution from diffractive events. Indeed separating the different components of MC models, one finds [3] that the models have very different descriptions of diffraction. PYTHIA6 has no hard diffraction included: the diffraction model used there produces no tracks for $p_{T}>3 \mathrm{GeV}$ and no diffractive events with $n_{\mathrm{ch}}>27$. PYTHIA8 and PHOJET both have a hard component to the diffraction extending up to higher multiplicities. The fraction of diffractive events vs $p_{T}$ is very roughly constant for these two models. All this is discussed more in Sect. 4.

Figure 5 summarizes the above observations. One can see that no MC model describes the data at low $n_{\mathrm{ch}}$ and low $p_{T}$, though AMBT1 and PYTHIA 8 are close to the data for $p_{T}>500 \mathrm{MeV}$. The deviations can be attributed to diffraction.

\section{Diffractive enhanced minimum bias studies}

Diffractive interactions, generally at low momentum transfer, can only be described by phenomenological models, and there is little consensus in the theoretical community as to which method is the best, leading to high uncertainty in model predictions at LHC energies. These uncertainty results to difficulties for tuning the non-diffractive portion, forcing the MC tunes to be made from phase-space areas where diffractive effects are negligible [9]. Meanwhile, the absence of color exchange in diffractive events leads to rapidity gaps, a characteristic which experimentalists can exploit. Therefore, to enhance the sample in diffraction, events were selected with a hit (in at least one MBTS cell) only on one, $z$-positive or 

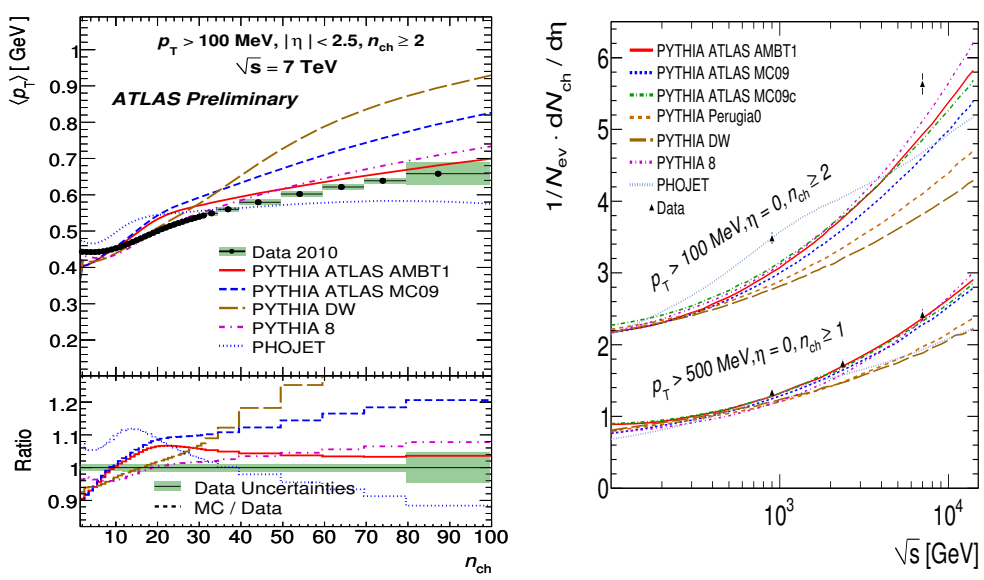

Figure 5: The left panel shows charged-particle averaged $p_{T}$ as function of multiplicity at $\sqrt{s}=7 \mathrm{TeV}$. The data are shown the same way as in Fig. 2. The right panel shows the central pseudorapidity density as a function of centre-of-mass energy.

$z$-negative, side of the MBTS. This requirement preferentially selects single and double-diffractive events. Double-diffractive events pass the selection if the mass of the diffractive system on one of the two sides is small enough that the particles all escape at $|\eta|>3.84$ and thus do not create a signal in the MBTS. The study uses the $7 \mathrm{TeV}$ data corresponding to an integrated luminosity of $\sim 23 \mu \mathrm{b}^{-1}$. The tracks selection criteria are similar to the above, while requiring $p_{T}>500 \mathrm{MeV}$, $\left|d_{0}^{B S}\right|<1.5 \mathrm{~mm},\left|z_{0}\right|<100 \mathrm{~mm}$, and $N_{\text {sct }} \geq 6$, where $z_{0}$ is defined with respect of the origin of the detector coordinate system. This event selection does not require a primary vertex, to avoid any bias at low track multiplicity. In addition a subset of events (the "single-sided" sample) was selected by vetoing events that have hits on both sides of the MBTS. The main systematic uncertainties were found to arise from beam backgrounds, data and MC agreement for the MBTS simulation and the tracking performance, and were carefully investigated [5].

Figure 6 shows the measured fraction $R_{s s}$ of the number of the "single-sided" events to the number of such events that have any hit in either side of the MBTS, compared to the Monte Carlo predictions. The measured value is $R_{s s}=$ $[4.52 \pm 0.02$ (stat.) \pm 0.61 (syst.) $] \%$. The predictions from PYTHIA6 MC09 $(4.01 \%)$ and PYTHIA8 $(5.11 \%$ ) agree well with the data while the PHOJET prediction $(2.83 \%)$ falls short by $70 \%$ corresponding to $2.9 \sigma$. The diffractive component needs to be increased from $20 \%$ to $30 \%$ in PHOJET to describe the data.

To investigate the event kinematics the $\eta, p_{T}$, multiplicity $n_{\text {trk }}$ and $\Delta \eta^{3}$ distributions were measured. As seen in [5], the the data $\eta$-spectrum is flat about

\footnotetext{
${ }^{3}$ Here $\Delta \eta$ is the absolute value of the difference in pseudorapidity between the edge of the MBTS detector that has no hit $\left(\eta_{M B T S}\right)$ and the track, i.e. $\Delta \eta=\left|\eta_{M B T S}-\eta\right|$ where $\eta_{M B T S}$ is +2.08 or -2.08 depending on which MBTS side did not have any hits.
} 
0.4, well modeled by PYTHIA8 and PHOJET, while underestimated by about $30 \%$ by PYTHIA6. Study of the $\Delta \eta$ spectrum shows [5] that at low $\Delta \eta$ all three generators describe the data well but at high $\Delta \eta$, where the diffractive processes dominate, PYTHIA6 underestimates the measurements.

Figures 7 and 8 show charged particle low-multiplicity and $p_{T}$ distributions, respectively. One can see that multiplicity distribution falls by five orders of magnitude over the range of 1 to 20 tracks. It is thus much steeper than that measured in the inclusive sample [3]. It is well modeled both by PYTHIA 8 and PHOJET while the PYTHIA6 predicts smaller multiplicities than the data apart from the tail, where the non-diffractive contribution plays a role. The $p_{T}$-spectra predicted by PHOJET is in excellent agreement with the data, while PYTHIA8 predicts slightly softer spectrum, and PYTHIA6 is even much softer.

In order to understand the origin of the features observed, a detailed study of the distributions for each generator was carried out with each of the subprocesses separately [5]. This confirms the importance of hard diffraction as incorporated in PYTHIA8 and indicated a need of an increase of the non-diffractive component both in PYTHIA8 and PHOJET relative to PYTHIA6.
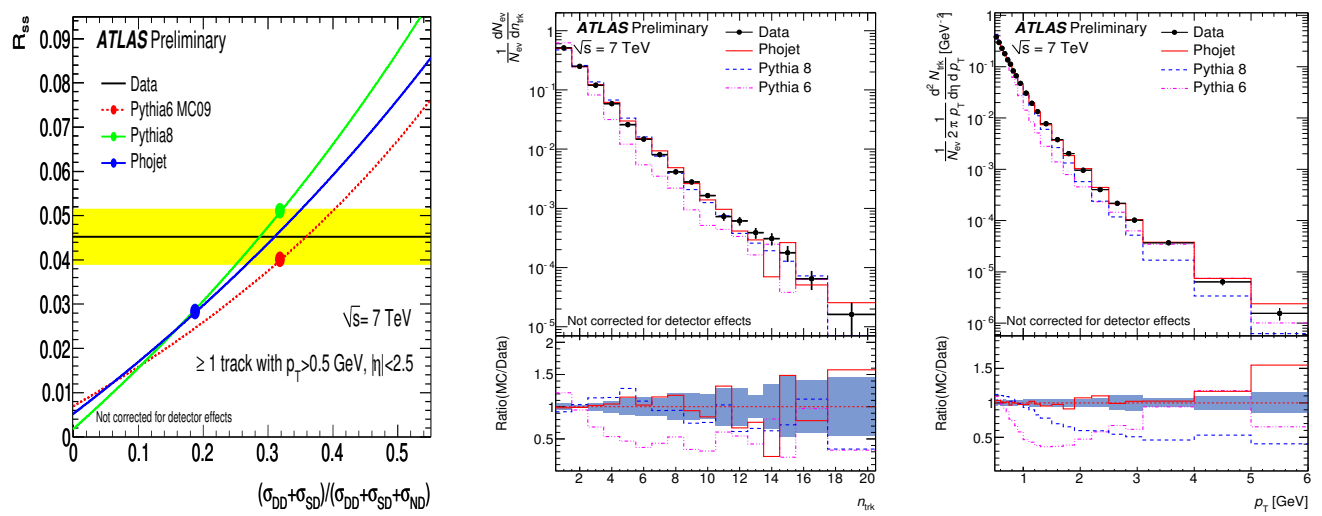

Figure 6: The ratio $R_{s s}$ of events with hits only on one side of the MBTS scintillators to events with any hits in the MBTS scintillators as a function of the diffractive contribution to the total inelastic cross section. The values for the cross sections used by these MC programs and the corresponding values of $R_{s s}$ are indicated by the solid circles. The ratio of the single to double diffractive cross sections is held fixed to the generator prediction.

Figure 7: The multiplicity track distributions for the single-sided MBTS requirement. The data are shown the same way as in Fig. 2.

Figure 8: The distributions of $p_{T}$ for the single-sided MBTS requirement. The data are shown the same way as in Fig. 2. 


\section{Conclusions}

Using first proton-proton data obtained at the LHC, soft QCD studies are carried out by the ATLAS experiment. Charged particle distributions are measured with the ATLAS detector at the center-of-mass energies $\sqrt{s}=0.9,2.36$ and $7 \mathrm{TeV}$. The measurements show good understanding of the detector and tracking performance. First measurements are performed for minimum bias events at all three energies and for diffractive events at ever large centre-of-mass energy. The results obtained are of great importance in our understanding of soft particle production processes and for improving phenomenological and Monte Carlo models.

\section{References}

[1] ATLAS Collaboration, Aad G. et al. // JINST. 2008. V.3. P.S08003.

[2] Kittel W., De Wolf E.A. Soft Multihadron Dynamics (World Scientific). 2005. $652 \mathrm{pp}$.

[3] ATLAS Collaboration. // ATLAS-CONF-2010-046. 2010.

[4] ATLAS Collaboration. // ATLAS-CONF-2010-047. 2010.

[5] ATLAS Collaboration. // ATLAS-CONF-2010-048. 2010.

[6] Bélanger-Champagne C. // This Workshop.

[7] ATLAS Collaboration, Aad G. et al. // Phys. Lett. B. 2010. V.688. P.21.

[8] Sjostrand T., Mrenna S., Skands P. Z. // JHEP. 2006. V.05. P.026.

[9] ATLAS Collaboration. // ATL-PHYS-PUB-2010-002. 2010.

[10] Marshall Z. // Conf. Note ATL-SOFT-PROC-2008-001. 2008.

[11] ATLAS Collaboration. // ATLAS-CONF-2010-031. 2010.

[12] Skands P. Z. // ArXiv:0905.3418 [hep-ph]. 2009.

[13] Albrow M. G. et al. // ArXiv:0610012 [hep-ph]. 2006.

[14] Sjostrand T., Mrenna S., Skands P. Z. // Comp. Phys. Comm. 2008. V.178. P.852.

[15] Egel R. // Z. Phys. C 1995. V.66. P.203. 